\title{
Evaluating the Potential of Third Generation Metagenomic Sequencing for the Detection of BRD Pathogens and Genetic Determinants of Resistance in Chronically ill Feedlot Cattle
}

\author{
Claire N. Freeman \\ University of Saskatchewan \\ Emily K. Herman \\ University of Alberta \\ Jennifer Abi Younes \\ University of Saskatchewan \\ Dana E. Ramsay \\ University of Saskatchewan \\ Nathan Erikson \\ University of Saskatchewan \\ Paul Stothard \\ University of Alberta \\ Cheryl Waldner ( $\nabla$ cheryl.waldner@usask.ca ) \\ University of Saskatchewan \\ Matthew G. Links \\ University of Saskatchewan \\ Simon J. G. Otto \\ University of Alberta
}

\section{Research Article}

Keywords:

Posted Date: February 25th, 2022

DOI: https://doi.org/10.21203/rs.3.rs-1319240/v1

License: @ (i) This work is licensed under a Creative Commons Attribution 4.0 International License. Read Full License 


\section{Abstract \\ Background.}

Bovine respiratory disease (BRD) is an important cause of morbidity and mortality and is responsible for most of the injectable antimicrobial use in the feedlot industry. Traditional bacterial culture can be used to diagnose BRD by confirming the presence of causative pathogens and to support antimicrobial selection. However, given that bacterial culture takes up to a week and early intervention is critical for treatment success, culture has limited utility for informing rapid therapeutic decision-making. In contrast, metagenomic sequencing has the potential to quickly resolve all nucleic acid in a sample, including pathogen biomarkers and antimicrobial resistance genes. In particular, third-generation Oxford Nanopore Technology sequencing platforms provide long reads and access to raw sequencing data in real-time as it is produced, thereby reducing the time from sample collection to diagnostic answer. The purpose of this study was to compare the diagnostic performance of nanopore metagenomic sequencing to traditional culture and sensitivity methods as applied to nasopharyngeal samples from segregated groups of chronically ill feedlot cattle, previously treated with antimicrobials for nonresponsive pneumonia or lameness.

\section{Results.}

BRD pathogens were isolated from most samples and a variety of different resistance profiles were observed across isolates. The sequencing data indicated the samples were dominated by Moraxella bovoculi, Mannheimia haemolytica, Mycoplasma dispar, and Pasteurella multocida, and included a wide range of antimicrobial resistance genes (ARGs), encoding resistance for up to seven classes of antimicrobials. Genes conferring resistance to beta-lactams were the most commonly detected, while the tetH gene was detected in the most samples overall. Metagenomic sequencing detected the BRD pathogens of interest more often than did culture, but there was limited concordance between phenotypic resistance to antimicrobials and the presence of relevant ARGs.

\section{Conclusions.}

Metagenomic sequencing can reduce the time from sampling to results, detect pathogens missed by bacterial culture, and identify genetically encoded determinants of resistance. Increasing sequencing coverage of target organisms will be an essential component of improving the reliability of this technology, such that it can be better used for the surveillance of pathogens of interest, genetic determinants of resistance, and to inform diagnostic decisions.

\section{Background}

Bovine respiratory disease (BRD) is an important cause of morbidity and mortality and is responsible for most of the injectable antimicrobial use in the feedlot industry [1]. BRD is a complex, multifactorial disease linked to a combination of viral and bacterial pathogens together with management and environmental factors impacting the host immune response [2]. Laboratory diagnostics are critical to inform appropriate antimicrobial use for disease management and therapy and to guide vaccination recommendations. Traditional bacterial culture with or without antimicrobial susceptibility testing (AST) can be used to confirm the presence of causative pathogens and to support antimicrobial selection. However, given that culture-based approaches can take up to a week to finalize and early intervention is critical to BRD treatment success [3], these approaches have limited utility for informing rapid therapeutic decisions. Recent WHO prescribing guidelines recommend basing all prescriptions for livestock on diagnostic test data [4].

In contrast to classical culture-based microbiology methods, molecular techniques including quantitative polymerase chain reaction (qPCR), whole genome sequencing (WGS), and metagenomic sequencing are gaining traction in diagnostic laboratories due to their comparatively faster speeds and potential for increased sensitivity. WGS generally requires that bacterial and viral pathogens are first isolated and cultured prior to nucleic acid extraction and sequencing, but can produce high resolution genomic information for serotyping, outbreak surveillance, and outbreak management [5-7]. qPCR and metagenomics differ from WGS in that they can both use DNA extracted directly from clinical samples, bypassing time-consuming culture-based steps. qPCR is already widely used in BRD diagnostics, and even exists in the form of commercially available kits (Pneumo4, DNA Diagnostic A/S, Risskov, Denmark). More recently, qPCR has been used to quantify the relative proportions of antimicrobial resistance genes (ARGs) in the nasopharyngeal microbiota of Canadian feedlot cattle [8, 9].

Metagenomics refers to either 1) amplicon sequencing, wherein a single conserved microbial gene is amplified and sequenced; or 2) shotgun metagenomics, wherein all DNA extracted from a sample is made available for sequencing. Although amplicon sequencing can offer improved species detection over shotgun metagenomics, it is not suitable for providing genetic information outside of taxonomic composition and relative abundance. Due to its untargeted nature, metagenomic sequencing has the potential to reveal anything encoded by nucleic acids present in the sample, including pathogen biomarkers, ARGs, and virulence genes [10]. In contrast to qPCR, the use of metagenomics in diagnostics has only recently been explored [11]. An examination of the diagnostic potential of sequencing technologies for BRD must evaluate its performance in tandem with existing gold standard methods, including bacterial culture and AST.

Concordance between culture, AST and WGS sequence data varies depending on the bacterial species, the tested antimicrobials, and the ARGs under investigation. For example, phenotypic resistance correlated highly (99\%) with the presence of known resistance determinants in isolates of nontyphoidal Salmonella from clinical and retail meat samples [12]. In contrast, the concordance between genotype and phenotype for antimicrobial-resistant Mannheimia haemolytica from cattle is reportedly lower, particularly for resistance to tilmicosin, tulathromycin, and florfenicol, which was largely as a result of ARGcontaining integrative and conjugative elements (ICE) [13]. Substantial genotype-phenotype discordance was likewise noted in a WGS study involving BRDassociated isolates (M. haemolytica, Pasteurella multocida and Histophilus somni) from beef and dairy calves [14]. 
Concordance between culture and metagenomic sequencing is decidedly less well characterized and faces many additional obstacles, including variable sequence quality, the absence of data related to transcriptional activity, and the presence of abundant host DNA [15]. In one study that compared 16S rRNA amplicon sequence data to conventional culture results from clinical samples, the authors reported a $91.8 \%$ and $52.8 \%$ concordance rate for culture-positive and culture-negative specimens, respectively [16]. Other studies have reported that measures of genotype-phenotype concordance are highly variable for the same sample depending on the bioinformatic analysis used [17].

With the exception of select metagenomic surveys that consider BRD-associated viral pathogens [18, 19], most BRD studies that employ metagenomic or whole-genome sequencing have used second-generation, short-read sequencing on Illumina, lon Torrent, and Roche 454 sequencing platforms [20, 21]. These platforms generally offer high sequence quality but have otherwise limited practicality for rapid diagnostics due to their lengthy library preparation protocols and the inaccessibility of data until after the sequencing run has finalized. In contrast, third-generation Oxford Nanopore Technology (ONT) platforms provide long reads and access to raw sequencing data in real-time as it is produced; this feature allows for bioinformatic analyses to be run simultaneously, including the identification of pathogens and ARGs. The feasibility of using an ONT device to produce sequencing data, assemble reads, and annotate the genomes of two M. haemolytica strains from pneumonic cattle was explored in a recent study [22]. The authors highlight the potential of nanopore technology to evaluate AMR, given that ARGs were identified from assemblies constructed with relatively few $(>5400)$ ultra-long reads and corresponded in most cases to phenotypic resistance.

Feedlot cattle that receive repeated treatments for BRD and fail to respond are generally relocated to designated "chronic pens" [23]. These animals are more likely than the general feedlot population to have been administered multiple classes of antimicrobials as part of their ongoing medical management [23]. The purpose of this study was to compare the diagnostic performance of nanopore metagenomic sequencing to traditional culture and sensitivity methods when applied to clinical nasopharyngeal samples from segregated groups of chronically ill feedlot cattle, primarily afflicted with nonresponsive pneumonia or lameness. The following effort to evaluate the use of direct DNA metagenomic sequencing to detect BRD pathogens and their resistance determinants thus targeted this subpopulation where both were expected to be present in greater relative proportions.

\section{Results}

Sequencing yielded $73.99 \mathrm{~Gb}$ in total (average $12.33 \pm 2.79$ per run) and produced 57.32 million reads (average $9.55 \pm 2.03$ per run). After quality filtering, each sample had an average of $1.93 \mathrm{~Gb}$ of data $( \pm 0.67 \mathrm{~Gb}$ ), while after host filtering, an average of 115.4 million bases remained per sample (full details in Supplementary Table 1, Additional File 1). Data have been deposited with the Short Read Archive (BioProject: PRJNA809384). The mean quality score of the post quality filtered reads was $13.7 \pm 0.3$. In general, the resulting sequences were mostly derived from host biomass, as an average of $94 \%$ of each sample was classified as Bos taurus by the bioinformatic pipeline.

\section{Culture and antimicrobial susceptibility}

Target BRD pathogens were isolated from 20 of 25 samples; $P$. multocida $(n=16)$ and $M$. bovis $(n=12)$ were the most commonly detected organisms. M. haemolytica was present in comparatively low numbers $(n=2)$, and $H$. somni was not isolated from any sample. Co-isolation of pathogens was common ( $n=$ 10). All recovered M. haemolytica and P. multocida isolates were susceptible to ceftiofur, danofloxacin, enrofloxacin and florfenicol. The MICs for all tested antimicrobial drugs are reported for each isolate in Table 1. 
Table 1

Minimum inhibitory concentrations for isolates recovered in this study.

\begin{tabular}{|c|c|c|c|c|c|c|c|c|c|c|c|c|c|c|c|c|}
\hline $\begin{array}{l}\text { Sample } \\
\text { No. }\end{array}$ & $\begin{array}{l}\text { BRD } \\
\text { Pathogen }\end{array}$ & $\begin{array}{l}\text { Culture } \\
\text { Result }\end{array}$ & AMP & TIO & CLIN* & DANO & ENRO & FLOR & GAM & GEN* & NEO* & PEN & SPECT & SUL* & TET & TIAM" \\
\hline 1 & M. bovis & & $\mathrm{N} / \mathrm{T}$ & $\mathrm{N} / \mathrm{T}$ & $\mathrm{N} / \mathrm{T}$ & $\mathrm{N} / \mathrm{T}$ & 0.12 & 4 & 128 & $\mathrm{~N} / \mathrm{T}$ & $\mathrm{N} / \mathrm{T}$ & 16 & $\mathrm{~N} / \mathrm{T}$ & $\mathrm{N} / \mathrm{T}$ & $\mathrm{N} / \mathrm{T}$ & $\mathrm{N} / \mathrm{T}$ \\
\hline 2 & M. bovis & & $\mathrm{N} / \mathrm{T}$ & $\mathrm{N} / \mathrm{T}$ & $\mathrm{N} / \mathrm{T}$ & $\mathrm{N} / \mathrm{T}$ & 0.12 & 4 & 64 & $\mathrm{~N} / \mathrm{T}$ & $\mathrm{N} / \mathrm{T}$ & 16 & $\mathrm{~N} / \mathrm{T}$ & $\mathrm{N} / \mathrm{T}$ & $\mathrm{N} / \mathrm{T}$ & $\mathrm{N} / \mathrm{T}$ \\
\hline 3 & P. multocida & Few & 0.25 & 0.25 & 32 & 0.12 & 0.12 & 0.50 & 16 & 4 & 64 & 0.12 & 128 & 512 & 8.0 & 64 \\
\hline 5 & M. bovis & & $\mathrm{N} / \mathrm{T}$ & $\mathrm{N} / \mathrm{T}$ & $\mathrm{N} / \mathrm{T}$ & $\mathrm{N} / \mathrm{T}$ & 8.00 & 8 & 512 & $\mathrm{~N} / \mathrm{T}$ & $\mathrm{N} / \mathrm{T}$ & 16 & $\mathrm{~N} / \mathrm{T}$ & $\mathrm{N} / \mathrm{T}$ & $\mathrm{N} / \mathrm{T}$ & $\mathrm{N} / \mathrm{T}$ \\
\hline 5 & P. multocida & $1+$ & 0.25 & 0.25 & 32 & 0.12 & 0.12 & 0.50 & 1 & 4 & 8 & 0.12 & 32 & 256 & 0.5 & 32 \\
\hline 6 & P. multocida & $2+$ & 0.50 & 0.25 & 32 & 0.12 & 0.12 & 0.50 & 2 & 4 & 64 & 1.00 & 32 & 512 & 8.0 & 32 \\
\hline 7 & M. bovis & & $\mathrm{N} / \mathrm{T}$ & $\mathrm{N} / \mathrm{T}$ & $\mathrm{N} / \mathrm{T}$ & $\mathrm{N} / \mathrm{T}$ & 4.00 & 4 & 256 & $\mathrm{~N} / \mathrm{T}$ & $\mathrm{N} / \mathrm{T}$ & 16 & $\mathrm{~N} / \mathrm{T}$ & $\mathrm{N} / \mathrm{T}$ & $\mathrm{N} / \mathrm{T}$ & $\mathrm{N} / \mathrm{T}$ \\
\hline 7 & P. multocida & $1+$ & 0.25 & 0.25 & 16 & 0.12 & 0.12 & 0.50 & 1 & 1 & 4 & 0.25 & 32 & 512 & 0.5 & 8 \\
\hline 8 & M. bovis & & $\mathrm{N} / \mathrm{T}$ & $\mathrm{N} / \mathrm{T}$ & $\mathrm{N} / \mathrm{T}$ & $\mathrm{N} / \mathrm{T}$ & 4.00 & 8 & 512 & $\mathrm{~N} / \mathrm{T}$ & $\mathrm{N} / \mathrm{T}$ & 16 & $\mathrm{~N} / \mathrm{T}$ & $\mathrm{N} / \mathrm{T}$ & $\mathrm{N} / \mathrm{T}$ & $\mathrm{N} / \mathrm{T}$ \\
\hline 8 & P. multocida & $1+$ & 0.25 & 0.25 & 32 & 0.12 & 0.12 & 0.50 & 1 & 2 & 8 & 0.12 & 32 & 256 & 0.5 & 16 \\
\hline 9 & M. bovis & & $\mathrm{N} / \mathrm{T}$ & $\mathrm{N} / \mathrm{T}$ & $\mathrm{N} / \mathrm{T}$ & $\mathrm{N} / \mathrm{T}$ & 4.00 & 4 & 512 & $\mathrm{~N} / \mathrm{T}$ & $\mathrm{N} / \mathrm{T}$ & 16 & $\mathrm{~N} / \mathrm{T}$ & $\mathrm{N} / \mathrm{T}$ & $\mathrm{N} / \mathrm{T}$ & $\mathrm{N} / \mathrm{T}$ \\
\hline 9 & P. multocida & $4+$ & 0.50 & 0.25 & 32 & 0.12 & 0.12 & 0.50 & 2 & 4 & 64 & 2.00 & 32 & 512 & 4.0 & 64 \\
\hline 10 & M. bovis & & $\mathrm{N} / \mathrm{T}$ & $\mathrm{N} / \mathrm{T}$ & $\mathrm{N} / \mathrm{T}$ & $\mathrm{N} / \mathrm{T}$ & 0.12 & 2 & 32 & $\mathrm{~N} / \mathrm{T}$ & $\mathrm{N} / \mathrm{T}$ & 16 & $\mathrm{~N} / \mathrm{T}$ & $\mathrm{N} / \mathrm{T}$ & $\mathrm{N} / \mathrm{T}$ & $\mathrm{N} / \mathrm{T}$ \\
\hline 10 & P. multocida & $1+$ & 0.25 & 0.25 & 32 & 0.12 & 0.12 & 0.50 & 1 & 4 & 16 & 0.25 & 32 & 512 & 0.5 & 32 \\
\hline 11 & M. bovis & & $\mathrm{N} / \mathrm{T}$ & $\mathrm{N} / \mathrm{T}$ & $\mathrm{N} / \mathrm{T}$ & $\mathrm{N} / \mathrm{T}$ & 2.00 & 2 & 512 & $\mathrm{~N} / \mathrm{T}$ & $\mathrm{N} / \mathrm{T}$ & 16 & $\mathrm{~N} / \mathrm{T}$ & $\mathrm{N} / \mathrm{T}$ & $\mathrm{N} / \mathrm{T}$ & $\mathrm{N} / \mathrm{T}$ \\
\hline 11 & P. multocida & $1+$ & 0.25 & 0.25 & 32 & 0.12 & 0.12 & 0.50 & 1 & 4 & 8 & 0.12 & 32 & 256 & 0.5 & 32 \\
\hline 12 & M. bovis & & $\mathrm{N} / \mathrm{T}$ & $\mathrm{N} / \mathrm{T}$ & $\mathrm{N} / \mathrm{T}$ & $\mathrm{N} / \mathrm{T}$ & 4.00 & 2 & 512 & $\mathrm{~N} / \mathrm{T}$ & $\mathrm{N} / \mathrm{T}$ & 16 & $\mathrm{~N} / \mathrm{T}$ & $\mathrm{N} / \mathrm{T}$ & $\mathrm{N} / \mathrm{T}$ & $\mathrm{N} / \mathrm{T}$ \\
\hline 12 & P. multocida & $3+$ & 4.00 & 0.25 & 32 & 0.12 & 0.12 & 0.25 & 1 & 4 & 64 & 16.0 & 32 & 512 & 4.0 & 64 \\
\hline 13 & M. bovis & & $\mathrm{N} / \mathrm{T}$ & $\mathrm{N} / \mathrm{T}$ & $\mathrm{N} / \mathrm{T}$ & $\mathrm{N} / \mathrm{T}$ & 0.50 & 4 & 512 & $\mathrm{~N} / \mathrm{T}$ & $\mathrm{N} / \mathrm{T}$ & 16 & $\mathrm{~N} / \mathrm{T}$ & $\mathrm{N} / \mathrm{T}$ & $\mathrm{N} / \mathrm{T}$ & $\mathrm{N} / \mathrm{T}$ \\
\hline 14 & M. bovis & & $\mathrm{N} / \mathrm{T}$ & $\mathrm{N} / \mathrm{T}$ & $\mathrm{N} / \mathrm{T}$ & $\mathrm{N} / \mathrm{T}$ & 0.12 & 4 & 128 & $\mathrm{~N} / \mathrm{T}$ & $\mathrm{N} / \mathrm{T}$ & 16 & $\mathrm{~N} / \mathrm{T}$ & $\mathrm{N} / \mathrm{T}$ & $\mathrm{N} / \mathrm{T}$ & $\mathrm{N} / \mathrm{T}$ \\
\hline 14 & P. multocida & $1+$ & 16.0 & 0.25 & 32 & 0.12 & 0.12 & 0.50 & 2 & 4 & 64 & 16.0 & 32 & 512 & 4.0 & 64 \\
\hline 17 & $\begin{array}{l}\text { M. } \\
\text { haemolytica }\end{array}$ & $1+$ & 0.25 & 0.25 & 8 & 0.12 & 0.12 & 1.00 & 16 & 2 & 4 & 0.25 & 32 & 512 & 0.5 & 16 \\
\hline 18 & P. multocida & $2+$ & 0.25 & 0.25 & 32 & 0.12 & 0.12 & 0.25 & 1 & 2 & 64 & 0.50 & 32 & 512 & 4.0 & 32 \\
\hline 19 & P. multocida & $4+$ & 0.25 & 0.25 & 32 & 0.12 & 0.12 & 0.50 & 1 & 2 & 8 & 0.25 & 16 & 256 & 0.5 & 32 \\
\hline 20 & P. multocida & $1+$ & 4.00 & 0.25 & 32 & 0.12 & 0.12 & 0.50 & 2 & 4 & 64 & 8.0 & 32 & 512 & 8.0 & 64 \\
\hline 23 & P. multocida & $1+$ & 0.25 & 0.25 & 32 & 0.12 & 0.12 & 0.50 & 1 & 2 & 8 & 0.25 & 32 & 256 & 0.5 & 32 \\
\hline 24 & $\begin{array}{l}\text { M. } \\
\text { haemolytica }\end{array}$ & $1+$ & 0.25 & 0.25 & 8 & 0.12 & 0.12 & 0.50 & 1 & 1 & 4 & 0.12 & 32 & 256 & 0.5 & 16 \\
\hline 24 & P. multocida & $1+$ & 0.25 & 0.25 & 32 & 0.12 & 0.12 & 0.50 & 1 & 2 & 8 & 0.12 & 32 & 256 & 0.5 & 32 \\
\hline 25 & M. bovis & & $\mathrm{N} / \mathrm{T}$ & $\mathrm{N} / \mathrm{T}$ & $\mathrm{N} / \mathrm{T}$ & $\mathrm{N} / \mathrm{T}$ & 0.12 & 4 & 512 & $\mathrm{~N} / \mathrm{T}$ & $\mathrm{N} / \mathrm{T}$ & 16 & $\mathrm{~N} / \mathrm{T}$ & $\mathrm{N} / \mathrm{T}$ & $\mathrm{N} / \mathrm{T}$ & $\mathrm{N} / \mathrm{T}$ \\
\hline 25 & P. multocida & $1+$ & 0.50 & 0.25 & 32 & 0.12 & 0.12 & 0.50 & 1 & 2 & 8 & 0.12 & 32 & 256 & 0.5 & 32 \\
\hline
\end{tabular}

\section{[Table 1 Legend]}

Samples were screened for the presence of BRD pathogens and recovered isolates were tested for sensitivity to antimicrobials via serial broth microdilution using the BOPO7F panel. MICs for each antimicrobial were compared against CLSI breakpoints (full details in Supplementary Table 2, Additional File 2. N/T indicates an isolate was not tested against a certain antimicrobial. Antimicrobials without established breakpoints for the target organisms are indicated with an asterisk.

\section{Bacterial and ARG abundance in the sequence data}

Moraxella bovoculi, M. haemolytica, Mycoplasma dispar, and P. multocida dominated most samples in both the number of reads and the number of bases attributed to these organisms (Fig. 1). P. multocida was the most abundant BRD pathogen detected in the metagenomic sequencing data, followed by $M$. haemolytica, M. bovis, and H. somni (Fig. 2). The only organism detected via metagenomic sequencing in every single sample was $M$. dispar. Regarding the abundance of BRD-associated organisms, $P$. multocida was present in 23 samples, $M$. haemolytica was present in nine samples, $H$. somni was present in 
seven samples, and $M$. bovis was present in ten samples (Table 2). In general, each sample was dominated by one or two species (typically $M$. dispar, $M$. bovoculi, or P. multocida) with minimal representation from other species (Fig. 1).

\section{[Figure 1]}

Table 2

Concordance between culture and sequencing results for each sample.

\begin{tabular}{|c|c|c|c|c|c|c|c|c|c|c|c|c|}
\hline \multirow[b]{2}{*}{ Sample } & \multicolumn{4}{|c|}{ Culture Result } & \multicolumn{4}{|c|}{ Number of Sequencing Reads } & \multicolumn{4}{|c|}{ Concordance between Methods } \\
\hline & $M H$ & $P M$ & $H S$ & $M B$ & $M H$ & $P M$ & $H S$ & $M B$ & $M H$ & $P M$ & $H S$ & $M B$ \\
\hline 1 & & & & + & & & & 8 & MATCH & MATCH & MATCH & MATCH \\
\hline 2 & & & & + & 6 & 1 & 2 & 10 & Seq & Seq & Seq & $\mathrm{MATCH}$ \\
\hline 3 & & Few & & & & 9 & & 5 & MATCH & MATCH & MATCH & Seq \\
\hline 4 & & & & & & 7 & & & MATCH & Seq & MATCH & $\mathrm{MATCH}$ \\
\hline 5 & & $1+$ & & + & & 49 & 11 & 344 & MATCH & MATCH & Seq & MATCH \\
\hline 6 & & $2+$ & & & 80 & 4,085 & 16 & 1 & Seq & MATCH & Seq & Seq \\
\hline 7 & & $1+$ & & + & & 1,436 & 3 & 1 & MATCH & MATCH & Seq & MATCH \\
\hline 8 & & $1+$ & & + & & 660 & & 4 & MATCH & MATCH & MATCH & MATCH \\
\hline 9 & & $4+$ & & + & 89 & 14,198 & 10 & 8 & Seq & MATCH & Seq & MATCH \\
\hline 10 & & $1+$ & & + & & 55 & & 4 & MATCH & MATCH & MATCH & MATCH \\
\hline 11 & & $1+$ & & + & & 209 & 1 & & MATCH & MATCH & Seq & Cult \\
\hline 12 & & $3+$ & & + & 24 & 3,582 & 593 & & Seq & MATCH & Seq & Cult \\
\hline 13 & & & & + & 3 & 255 & & & Seq & Seq & MATCH & Cult \\
\hline 14 & & $1+$ & & + & & 33 & & & MATCH & MATCH & MATCH & Cult \\
\hline 15 & & & & & & & & & MATCH & MATCH & MATCH & MATCH \\
\hline 16 & & & & & & 2 & & & MATCH & Seq & MATCH & MATCH \\
\hline 17 & $1+$ & & & & 324 & 3 & 48 & & MATCH & Seq & Seq & MATCH \\
\hline 18 & & $2+$ & & & & 813 & & & MATCH & MATCH & MATCH & MATCH \\
\hline 19 & & $4+$ & & & 1 & 5,739 & 1 & 6 & Seq & MATCH & Seq & Seq \\
\hline 20 & & $1+$ & & & & 1,339 & 1 & & MATCH & MATCH & Seq & $\mathrm{MATCH}$ \\
\hline 21 & & & & & 34 & 520 & 1 & & Seq & Seq & Seq & MATCH \\
\hline 22 & & & & & 65 & 67 & & & Seq & Seq & MATCH & MATCH \\
\hline 23 & & $1+$ & & & 381 & 2,306 & & 4 & Seq & MATCH & MATCH & Seq \\
\hline 24 & $1+$ & $1+$ & & & 7,926 & 10,712 & 9 & 15 & MATCH & MATCH & Seq & Seq \\
\hline 25 & & $1+$ & & + & 27 & 1,222 & & 5 & Seq & MATCH & MATCH & MATCH \\
\hline
\end{tabular}

[Table 2 Legend] The columns under "culture result" indicate the semiquantitative abundance of each recovered isolate (MH = Mannheimia haemolytica, $\mathrm{PM}=$ Pasteurella multocida, $\mathrm{HS}=$ Histophilus somni, $\mathrm{MB}=$ Mycoplasma bovis). The columns under "Number of sequencing reads" indicate the number of sequencing reads that were classified as each of the four target pathogens, regardless of length. The columns under "concordance between methods" indicates whether the two approaches yielded the same answer ("MATCH"), Sequencing detected the organism when culture did not ('Seq"), or culture detected the organism when sequencing did not ("Cult").

Twenty-six different ARGs encoding resistance to aminoglycosides (aph(3')-la, aadA31, aph(3")-Ib, aph(6)-Id), beta-lactams (blaBRO, blaCARB, blaROB), macrolides (erm35, ermC, mphE), phenicols ( $\mathrm{cm} x$, floR), linocosamides (IunC, IsaB), tetracyclines (tet34, tetB, tetH, tetQ, tetW, tet $X$, tetY), and trimethoprim (dfrA14) were detected in the metagenomic sequences across 13 unique samples. Genes conferring resistance to the beta-lactam drug class were the most commonly detected and were found in eight different samples. tetH was the most frequently detected resistance gene overall $(n=6)$ followed by $m p h E(n=5)$, blaROB-5 and aadA37 $(\mathrm{n}=4)$. In 4 of the 8 samples where neither $P$. multocida nor $M$. haemolytica were isolated via culture, ARGs were detected in the sequence data, while in in the 18 samples where these organisms were detected, 8 samples had ARGs in the sequence data.

[Figure 2]

\section{Concordance between culture and sequencing}


In general, more BRD pathogens were detected via sequencing than by culturing (Table 2). For five samples, the presence or absence of all four BRD pathogens as determined by culturing and sequencing were in perfect agreement. The rate of concordance between culture and sequencing was highest for the detection of P. multocida, where the two approaches yielded the same result in $72 \%$ of samples (Cohen's K: 0.27 ). For the detection of $M$. bovis and $M$. haemolytica, the culture and sequence data yielded the same result in $64 \%$ and $60 \%$ of samples, respectively (Cohen's $\mathrm{k}$ : $0.28,0.17$ ). As $H$. somni was not detected by culture in any sample, Cohen's $\mathrm{k}$ could not be calculated. For those samples where the two diagnostic approaches did not match, sequencing detected M. haemolytica, P. multocida, and H. somni when culture did not. M. bovis was likewise detected via sequencing in five samples where none was recovered by culture, but the reverse was true for four additional samples where the organism was cultured but no sequencing reads were detected.

Concordance between the AST results for the Gram-negative pathogens and the ARGs detected via metagenomic sequencing was generally low (Table 3). Of the six isolates exhibiting resistance to beta-lactams (ampicillin, ceftiofur or penicillin), only three samples contained relevant ARGs in the sequencing data (blaCARB-5, blaROB-2,4,5); conversely, five samples without phenotypic resistance to beta-lactams had ARGs associated with resistance to this drug class. Only one of two isolates that demonstrated resistance to macrolides (gamithromycin and tulathromycin) had macrolide resistance genes ( $m p h E$ ), whereas five other samples with no phenotypic resistance to macrolides had detectable resistance genes in the sequence data ( $m p h E$, erm35, ermC). Similarly, ARGs encoding resistance to aminoglycosides and tetracyclines (tetH, tet $X$, tet34) were detected in only $33 \%(5 / 15)$ and two of three isolates with phenotypic resistance to at least one drug in these classes, respectively. Samples without phenotypic resistance to aminoglycosides $(n=2)$ or tetracycline $(n=6)$ nevertheless had ARGs associated with resistance to these classes.

The recovered $M$. bovis isolates were highly resistant to the antimicrobials they were tested against, and elevated MICs were particularly notable for gamithromycin, tildipirosin, tilmicosin and tylosin. WGS revealed that three M. bovis isolates had cytosine-to-thymine transitions at positions 683 and 798 of the 23S rRNA gene, which could confer resistance to macrolides. However, several isolates that did not have a SNP at these positions also demonstrated elevated MICs, and therefore agreement between the sequence and AST data was low for this organism.

\section{Discussion}

The upper respiratory tracts of the cattle sampled in this study were dominated by Moraxella bovoculi, Mycoplasma dispar, and Pasteurella multocida, a finding consistent with other studies of the bovine respiratory microbiome in healthy $[24,25]$ and unhealthy $[26,27]$ feedlot cattle in North America. Although M. bovoculi is frequently found in the bovine respiratory tract, it is more commonly associated with bovine keratoconjunctivitis [28], though the species from these two niches are genetically distinct [29]. M. bovoculi and the broader Moraxella genus do not have a recognized role in bovine respiratory disease. In contrast, both Mycoplasma dispar and $P$. multocida are not only frequently detected in the nasopharynx as commensal microbiota, but are known opportunistic pathogens that actively contribute to respiratory disease [30-32]. The frequent co-occurrence of these species is remarkable in that several studies suggest synergism between Mycoplasma sp. and P. multocida [31, 33, 34] as well as a possible initiative role of M. dispar in the development of respiratory tract disease in dairy cattle [35-38]. While the pathogenicity of $M$. dispar is well described in dairy calves, particularly in countries free of $M$. bovis, it has received little attention in BRD feedlot studies. However, this could be a consequence of the challenges associated with the fastidious nature of this organism rather than its potential importance in BRD [38]. In this regard, culture-independent approaches like metagenomic sequencing offer the opportunity to expand pathogen detection beyond the more typical predetermined BRD pathogens of interest (M. haemolytica, P. multocida, H. somni) for which culturebased techniques are well-established.

In this study, P. multocida was the most abundant species in one third of all samples, representing a larger fraction of reads than what has been previously reported in other metagenomic studies on Canadian feedlots. This abundance can be partially explained by the population of chronically diseased animals sampled in this study. Unlike the primary insulting pathogens identified from acutely ill cases of BRD during the early stages of infection, $P$. multocida is an opportunist, and is more often implicated in cattle with subacute or chronic pneumonia [31,39,40]. Chronically diseased cattle are less likely to be shedding high numbers of pathogens as in acute cases. Therefore, the relative abundance of bacteria in this study's chronically ill cattle was expected to be different compared to other studies which describe apparently healthy animals or acute cases of BRD.

The multifactorial nature of treatment failure (due to host, pathogen, drug, drug administration, and environmental factors) [23], as well as the slightly mixed population of cattle considered unresponsive to treatment, may further account for the pathogens observed. While most cattle in the chronic pens sampled in this study were considered chronic pneumonias, cattle with nonresponsive lameness were also present. In additional to naturally harbouring a different respiratory microbiome, chronically lame cattle likely received different antimicrobial protocols to their respiratory counterparts.

The differences seen between the dominant organisms in this study and those of previously published studies could potentially also be due to the relatively limited sequencing coverage of bacterial genomes in these samples. However, it is worth noting that most previous studies exploring the nasal microbiome have relied on sequencing one or two hypervariable regions of the 16S rRNA gene, and oftentimes this approach cannot be reliably used to classify sequences at the species or subspecies level [41].

The ARGs detected in these samples were associated with resistance to drugs commonly used in the cattle industry [1], including macrolides (erm35, erm $C$, $m p h E)$, phenicols (florR, $c m x)$, and tetracyclines (tet34, tetB, tet $H$, tetQ, tet $W$, tet $X$, tet $Y$ ). While these genes were detectable in the sequence data, relatively short fragment sizes and limited coverage in this preliminary proof of concept trial often impeded the classification of ARG-containing reads beyond the level of phylum. The ARGs detected in this study are similar to those of previous DNA-based surveys of the bovine respiratory tract [8,9,21] and even those of other bovine microbiome sites [42]. In particular, tet(H), the ARG present in the greatest number of samples, has been detected in integrative and conjugative element (ICE)-containing strains of M. haemolytica, P. multocida, and H. somni isolated from confirmed BRD cases [43].

The other tetracycline resistance genes (tet34, tet $B$, tetQ, tetW, tet $X$, tet $Y$ ) detected in this study were found in fewer samples and with the exception of tet( $B$ ), are not known to occur in pathogens of the bovine respiratory system in feedlot cattle. Tet( $B$ ), which encodes a tetracycline efflux pump, has been found in 
E.coli isolated from bovine feces [44], feedlot fecal composite samples [45], feedlot wastewater lagoons [46], and P. multocida isolates derived from cattle [47]. Tet $(W)$, tet $(Q)$, and tet $(X)$ have been detected in bronchoalveolar lavage and deep nasopharyngeal swab samples collected from feedlot cattle, but it was not evident that these genes were present in BRD pathogens rather than environmental or commensal populations $[8,21,48,49]$.

The macrolide phosphotransferase gene, $m p h E$, was also detected in several samples. This gene has been found in M. haemolytica isolates derived from pneumonic bovine lung tissue [22,50], and bronchoalveolar lavage samples collected from BRD cattle confirmed to have died of BRD [21]. This gene is frequently detected with $m s r E$, as the two occur together in an operon on the ICE, ICEPmu1, which has been found in several members of the Pasteurellaceae family [50,51]; however, msrE was not detected in any sample in this study. The aadA31 gene was also detected in several samples and encodes a spectinomycin/streptomycin adenylyltransferase. This gene has previously been detected in $P$. multocida and $H$. somni recovered from confirmed BRD mortalities where it was located inside a variant of the ICE, ICEMh1 [52]. A notable unifying theme among the most abundant ARGs in these samples is that many have been detected on ICE found in BRD-associated pathogens. The presence of these ARGs on ICE could explain why they are present in the higher abundance, but additional sequencing coverage would be necessary to confirm this.

Most M. bovis isolates recovered in this study had high MICs for gamithromycin, tildipirosin, tilmicosin and tylosin. While WGS demonstrated that some of these isolates had point mutations in the 23S rRNA gene that could be responsible for the resistant phenotype, this genetic pattern was not present in all resistant isolates and differed from the SNP signature of previously reported resistant $M$. bovis isolates in other studies [53, 54]. Antimicrobial resistance in Mycoplasma spp. differs from that of the other BRD pathogens, as members of this genus typically achieve resistance through point mutations rather than through the acquisition of ARGs [54, 55]. In this study, M. bovis reads were detected in 13 samples, ranging in abundance from 1 to 344 reads; the detection of genetic determinants of resistance in the metagenome was therefore not feasible for $M$. bovis, due to the low sequencing coverage. While the accurate detection of SNPs in metagenomes has been previously documented [56], it requires high sequence coverage of target genomes (typically in excess of 100x).

For M. haemolytica, P. multocida and H. somni, sequencing detected the presence of the pathogens more frequently than did culture. Indeed, DNA-based approaches are often better equipped to identify the presence of pathogens, as they can detect bacteria that are growth-inhibited or dead following antimicrobial therapy [57]. A comparison of these methods is more complex for $M$. bovis, insofar that the pathogen was recovered from culture but not the sequence data on four occasions. Given that the genome of $M$. bovis is approximately one-third of the length of the other BRD pathogens, it would have a slightly higher limit of detection on a per-cell basis and might therefore have been missed by metagenomic sequencing. Further and owing to its lack of cell wall, M. bovis may have been absent from some samples due to inherent incompatibilities with sample processing. This is probably unlikely given the comparatively large representation from other Mycoplasma spp. detected in sequence, such as $M$. dispar.

\section{Conclusion}

The benefits of metagenomic sequencing compared to traditional laboratory methods are severalfold and include the reduced time from sampling to results; the potential to detect pathogens missed by bacterial culture; and the ability to simultaneously identify genetically encoded determinants of resistance. In this study, metagenomic sequencing detected the BRD pathogens of interest more often than did culture, but there was limited concordance between phenotypic resistance to antimicrobials and the presence of relevant ARGs. This finding is likely due to limited coverage of the target organisms in response to the overabundance of bovine-derived sequences. As sequencing depth of relevant (i.e., non-host) sequences increases, so too will the coverage of BRD pathogens and their genetic determinants of resistance. This goal can be achieved by focusing future work on the preferential enrichment of target sequences in the following ways: 1) optimization of host DNA depletion protocols to reduce the amount of bovine DNA in samples prior to extraction; and 2) targeted DNA enrichment strategies, including bait capture, to reduce the amount of off-target sequence post-extraction. Increasing target sequencing coverage will be an essential component of improving the reliability of this technology, such that it can be better used for the surveillance of pathogens of interest and genetic determinants of resistance, and to inform diagnostic decisions.

\section{Methods}

\section{Animals}

Twenty-five mixed-breed steers from two chronic pens of a commercial feedlot in Saskatchewan were enrolled in the investigation. The holding capacity of the feedlot was approximately 25,000 cattle. Samples were collected once in the summer of 2020, near time of finishing, with cattle weights nearing 1,400 pounds. The two cohorts were purposely selected due to their history of chronic respiratory disease or lameness that was non-responsive to medical management, with most animals having received two or more antimicrobial treatments.

Processing and management of feedlot cattle was performed using standard industry protocols and included on-arrival vaccination for pathogens associated with BRD and clostridial disease, a pour-on anthelminthic drug, a hormone growth implant, and metaphylactic administration of tulathromycin (Draxxin, Zoetis Inc., Florham Park, New Jersey, USA). Cattle were fed a diet that met the National Research Council requirements for beef cattle throughout the feeding period. Experienced pen checkers observed animals daily for signs of clinical illness. Animals exhibiting such signs were treated following disease-specific antimicrobial protocols.

\section{Sample Collection}

Sampling was performed during one visit by one veterinarian experienced in collecting nasal and deep nasopharyngeal swabs. To facilitate sample collection, cattle were restrained in a hydraulic chute with neck extenders placed to stabilize the animal's head. The nares were wiped clean of debris with single use paper towels. Next, the first of three double-guarded nasopharyngeal swabs were collected. The $79 \mathrm{~cm}$, three-pieced culture swab (Continental Plastic Corp., Delevan, Wisconsin, USA) was advanced into the ventral meatus of the nostril until approximately $2 \mathrm{~cm}$ ventral from the medial canthus of the eye. The inner 
sheath was advanced through the outer guard to allow for the swab tip to be extended and vigorously rotated against the pharyngeal mucosa for at least ten seconds. The swab tip was withdrawn into the guarded sheaths prior to removal from the nostril. The swab tip was then cut and placed in $3 \mathrm{ml}$ of Amies transport medium. The procedure was repeated using alternating nostrils for a total of three times per calf and these deep nasopharyngeal (DNP) samples were pooled into the same vial of media. The samples were placed on ice and transported back to the University of Saskatchewan on the day of collection.

\section{Sample Processing}

The swabs and media were vortexed for one minute to release biomass from the swab into the transport medium. After vortexing, the swabs were removed. Two $\mathrm{ml}$ of media were centrifuged at $9,000 \mathrm{xg}$ for five minutes to pellet all biomass, and then $1800 \mathrm{ul}$ of supernatant were decanted to bring the total volume to $200 \mathrm{ul}$. The pellet was resuspended in the remaining supernatant and host depleted using the MolYsis Basic5 kit according to manufacturer's instructions (Molzym, Bremen, Germany). The resulting host depleted biomass pellet was then used in a total nucleic acid extraction with the MasterPure Complete DNA and RNA Purification Kit according to the manufacturer's instructions (Lucigen, Middleton, Wisconsin, USA). Extracted DNA was quantified using the Qubit ${ }^{\mathrm{TM}}$ 1X dsDNA High Sensitivity Assay Kit (Invitrogen, Carlsbad, California, USA) according to manufacturer's specifications and then kept at $4^{\circ} \mathrm{C}$ until library preparation.

\section{Culture and Antimicrobial Sensitivity Testing}

Pooled, vortexed media was processed the same day for the isolation of Mannheimia haemolytica, Pasteurella multocida, Histophilus somni, and Mycoplasma bovis. One chocolate agar and one blood agar plate was inoculated with $10 \mu$ of sample and incubated at $35 \otimes C$ in $\mathrm{CO}_{2}$ for 48 hours. At 24 and 48 hours, plates were examined for growth and bacterial colonies suspected of being $M$. haemolytica, $H$. somni, or $P$. multocida were confirmed using a MALDI-TOF MS Microflex LT instrument and MALDI Biotyper software (Bruker Corporation, Billerica, Massachusetts, USA). BRD pathogens isolated from samples were tested for antimicrobial susceptibility via serial broth microdilution using a commercially available panel (BOPO7F). Minimum inhibitory concentrations (MICs) for each antimicrobial were compared against breakpoints designated by the Clinical and Laboratory Standards Institute [58]. As there are no approved MIC breakpoint values for Mycoplasma bovis, antimicrobial susceptibility for $M$. bovis was assessed according to existing recommendations [59].

\section{Library preparation and sequencing}

Extracted sample DNA was prepared for sequencing using the Oxford Nanopore SQK-PBK004 kit (Oxford Nanopore, Oxford, England). After end repair and barcode ligation, sample DNA was amplified with LongAmp Hot Start Taq DNA Polymerase (New England Biolabs, Ipswich, Massachusetts, USA) using the

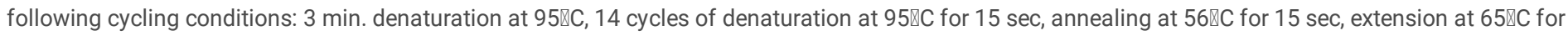
$6 \mathrm{~min} 40 \mathrm{sec}$, and a final extension step at $65 \mathrm{C}$ for $6 \mathrm{~min}$. The final fragment size of each library was determined with a Genomic DNA ScreenTape on a 4150 TapeStation System (Agilent, Santa Clara, California, USA) and then pooled in equimolar concentrations in groups of 4 to 5 samples. Sequencing was performed on a GridION Mk1 for 72 hours per run using FLO-MIN106 R9.4.1 flow cells.

\section{Bioinformatic Analysis}

Basecalling of raw signal was performed using Guppy (v4.0). After basecalling, terminal adapters and internal adapters in split reads were trimmed with Porechop v0.2.4 [60]. NanoFilt v2.6.0 was then used to remove any reads shorter than $100 \mathrm{bp}$, and sequence statistics were calculated using NanoStat v1.5.0 [61].

To classify host and non-host reads, Kraken2 v2.0.8-beta [62] was used with a confidence threshold of 0.1 and a custom database. The classification database included all complete genomes in RefSeq for the bacterial, viral, and archaeal domains, and all RefSeq plasmid nucleotide sequences as of October 17, 2020. The Bos taurus reference genome assembly ARS-UCD1.2_Btau5.0.1Y, which is available at http://www.1000bullgenomes.com/ [63] was also added to the classification database. Following classification, reads were split into two datasets using the KrakenTools v1.0 utility extract_kraken_reads.py: those assigned to the $B$. taurus taxid 9913 (host dataset), and those that were classified as any other taxid or were unclassified (non-host dataset).

The custom script kmer_filter.py was used to retrieve chimeric reads (an artefactual fusion of $B$. taurus and bacterial sequence) from the host dataset. This script detects reads where $<75 \%$ of the Kraken2-defined $k$-mers are not classified as $B$. taurus relative to the total number of $k$-mers, excluding $k$-mers classified as root (taxid 1), cellular organisms (taxid 131567), or unclassified (taxid 0). Reads that meet this criterion were then removed from the host dataset and added back to the non-host dataset in order to allow for possible cases of chimeric data (host + microbial). NanoStat was used to generate sequence statistics for the non-host dataset.

Bracken v2.5 [64] was used to generate taxonomic abundance estimates using the output of Kraken2. Bracken was run with default options, and reads assigned to taxid 9913 (i.e. potentially chimeric reads) were filtered using the KrakenTools script filter_bracken.out.py. Krona v2.7.1 [65] was used to create interactive Krona-style plots for visualization of the Kraken2 results.

Both Abricate v1.0.1 [66] and AMRFinderPlus v3.9.8 [67] were used to identify ARGs in the non-host reads using the NCBI Bacterial Antimicrobial Resistance Reference Gene Database (PRJNA313047, version 2020-12-17). The minimum percent identity and percent coverage for ARG detection by Abricate was kept at the default value of $80 \%$ for both, while a lower threshold of $60 \%$ was used for AMRFinderPlus, as AMRFinderPlus tends to be more stringent in reporting ARGs. The -plus option was also used with AMRFinderPlus, which directs the program to also search for genes involved in virulence, biocide, heat, metal, and acid resistance.

For samples where metagenomic contigs could be assembled, Abricate and AMRFinderPlus were also used to detect ARGs in contigs and any unassembled reads, after mapping the non-host reads to the metagenomic contigs with Minimap2 v2.13-r850 [68] (with pre-set input option map-ont) and filtering unmapped reads with Samtools v.1.10 [69]. 
To detect plasmid fragments in the non-host dataset, reads were used as queries in BLASTN searches against the PLSDB plasmid database (v2020_11_19), a curated database of bacterial plasmids from NCBI [70]. BLAST + v2.10.0 [70, 71] was used with an E-value cutoff of 1E-6, a minimum percent identity cutoff of $80 \%$, and a minimum query coverage per HSP cutoff of $80 \%$. Due to the relatively small BLAST database, shorter reads, and high plasmid sequence similarity, the custom script parse_plasmids.py was used to parse the output. This script generates a file with the top hit for each read and taxonomic annotation when the bitscore of the top hit is at least 10 points higher than the next hit from a different organism.

Metagenomic contig assembly of non-host reads was performed with Flye v2.8.1-bl676 [72, 73]. The -meta flag was used to indicate metagenome assembly mode, which is designed for highly non-uniform coverage and is sensitive to underrepresented sequence at low ( $<2 \mathrm{X})$ coverage. The input reads were specified as -nano-raw, which supports ONT reads that have not undergone error correction.

The Contig Annotation Tool (CAT) v5.2.3 [74] was used for contig classification with the 2020-06-18 version of the CAT database. Default parameters were used, with the addition of the -only-official flag when running the add_names module. This reports only classifications with the standard taxonomic levels (e.g., superkingdom, phylum, etc.), allowing the program to output a summary of the classification results.

The results of the workflow analyses were then parsed by a custom script, summarize_results.py. This script creates an Excel (.xslx) file that summarizes sequence statistics, ARGs, metagenomic assembly statistics, taxonomy results, and plasmid results. It adds further value to the output files generated by the programs in the workflow, as it also calculates and reports the percent non-host sequence and annotates ARGs with Kraken2 and plasmid taxonomy information where appropriate.

\section{Whole genome sequencing of Mycoplasma bovis}

As a comparison to metagenomic sequence data, Mycoplasma bovis isolates recovered from the DNP samples were used in a DNA isolation with the MasterPure Complete DNA and RNA Purification Kit according to the manufacturer's instructions (Lucigen, Middleton, Wisconsin, USA). Resulting DNA was prepared for sequencing using the ONT Ligation Sequencing Kit (SQK-LSK109) and run on one R9.4.1 FLO-MIN106 flow cell for 72 hours using a GridION Mk1. Resulting raw signal (fast5) files were basecalled using Guppy (v4.2.0; ONT) and assembled using Flye Assembler v2.8.1-bl676. Final consensus sequences were generated after polishing by Medaka (v.0.10.0; ont). The presence of single nucleotide polymorphisms (SNPs) in M. bovis genomes conferring antimicrobial resistance were predicted by Snippy (v.2.6) [75].

\section{Declarations}

\section{Ethics approval and consent to participate}

All experiments were performed in accordance with relevant guidelines and regulations. The animal use protocol was reviewed and approved by the Animal Research Ethics Board at the University of Saskatchewan and followed the principles established by the Canadian Council on Animal Care (Animal Use Protocol \# 20190069). All methods are in accordance with the ARRIVE guidelines for the reporting of animal experiments.

\section{Consent for publication}

Not applicable.

\section{Availability of data and materials}

The datasets generated in this study are available in the NCBI Sequence Read Archive (SRA) under BioProject: PRJNA809384 http://www.ncbi.nlm.nih.gov/bioproject/809384

\section{Competing interests}

The authors declare that they have no competing interests.

\section{Funding}

Funding for this study was provided by the Beef Cattle Research Council under project number BCRC_POC.04.18. This agency played no role in the design of the study.

\section{Authors' contributions}

CF designed the sample processing workflow, carried out the sample processing and sequencing, and drafted the manuscript. EH, PS and ML designed the bioinformatic pipeline, analysed all samples, and provided manuscript review. JAY and NE collected samples and provided manuscript review. DR and SO provided manuscript review. CW acquired funding, designed the study, and provided manuscript review.

\section{Acknowledgements}

The authors would like to thank Emmanuel Donbraye for his help with sample processing and Dhinesh Periyasamy for his invaluable guidance on sequencing library preparation.

\section{References}


1. Brault SA, Hannon SJ, Gow SP, Warr BN, Withell J, Song J, et al. Antimicrobial Use on 36 Beef Feedlots in Western Canada: 2008-2012. Front Vet Sci. 2019 Oct 17;6.

2. Griffin D, Chengappa MM, Kuszak J, McVey DS. Bacterial Pathogens of the Bovine Respiratory Disease Complex. Vet Clin North Am Food Anim Pract. 2010 Jul 1;26(2):381-94.

3. Wolfger B, Timsit E, White BJ, Orsel K. A Systematic Review of Bovine Respiratory Disease Diagnosis Focused on Diagnostic Confirmation, Early Detection, and Prediction of Unfavorable Outcomes in Feedlot Cattle. Vet Clin North Am Food Anim Pract. 2015 Nov;31(3):351-65.

4. Aidara-Kane A, Angulo FJ, Conly JM, Minato Y, Silbergeld EK, McEwen SA, et al. World Health Organization (WHO) guidelines on use of medically important antimicrobials in food-producing animals. Antimicrob Resist Infect Control. 2018 Dec 17;7(1):7.

5. Ibrahim GM, Morin PM. Salmonella Serotyping Using Whole Genome Sequencing. Front Microbiol. 2018 Dec 13;9(DEC):2993.

6. Besser JM, Carleton HA, Trees E, Stroika SG, Hise K, Wise M, et al. Interpretation of Whole-Genome Sequencing for Enteric Disease Surveillance and Outbreak Investigation. Foodborne Pathog Dis. 2019 Jul 1;16(7):504-12.

7. Harrison EM, Paterson GK, Holden MTG, Larsen J, Stegger M, Larsen AR, et al. Whole genome sequencing identifies zoonotic transmission of MRSA isolates with the novel mecA homologue mecC. EMBO Mol Med. 2013 Apr 25;5(4):509-15.

8. Guo Y, McMullen C, Timsit E, Hallewell J, Orsel K, van der Meer F, et al. Genetic relatedness and antimicrobial resistance in respiratory bacteria from beef calves sampled from spring processing to 40 days after feedlot entry. Vet Microbiol. 2020 Jan 1;240:108478.

9. Holman DB, Timsit E, Booker CW, Alexander TW, DB H, E T, et al. Injectable antimicrobials in commercial feedlot cattle and their effect on the nasopharyngeal microbiota and antimicrobial resistance. Vet Microbiol. 2018 Feb 1;214(November 2017):140-7.

10. Zhou M, Wu Y, Kudinha T, Jia P, Wang L, Xu Y, et al. Comprehensive Pathogen Identification, Antibiotic Resistance, and Virulence Genes Prediction Directly From Simulated Blood Samples and Positive Blood Cultures by Nanopore Metagenomic Sequencing. Front Genet. 2021 Mar 24;12:620009.

11. Chiu CY, Miller SA. Clinical metagenomics. Nat Rev Genet. 2019;20(6):341-55.

12. McDermott PF, Tyson GH, Kabera C, Chen Y, Li C, Folster JP, et al. Whole-Genome Sequencing for Detecting Antimicrobial Resistance in Nontyphoidal Salmonella. Antimicrob Agents Chemother. 2016 Sep 1;60(9):5515-20.

13. Snyder ER, Savitske BJ, Credille BC. Concordance of disk diffusion, broth microdilution, and whole-genome sequencing for determination of in vitro antimicrobial susceptibility of Mannheimia haemolytica. J Vet Intern Med. 2020 Sep 7;34(5):2158-68.

14. Owen JR, Noyes N, Young AE, Prince DJ, Blanchard PC, Lehenbauer TW, et al. Whole-Genome Sequencing and Concordance Between Antimicrobial Susceptibility Genotypes and Phenotypes of Bacterial Isolates Associated with Bovine Respiratory Disease. G3 Genes|Genomes|Genetics. 2017 Sep 1;7(9):3059-71.

15. Pereira-Marques J, Hout A, Ferreira RM, Weber M, Pinto-Ribeiro I, Van Doorn LJ, et al. Impact of host DNA and sequencing depth on the taxonomic resolution of whole metagenome sequencing for microbiome analysis. Front Microbiol. 2019;10(JUN).

16. Abayasekara LM, Perera J, Chandrasekharan V, Gnanam VS, Udunuwara NA, Liyanage DS, et al. Detection of bacterial pathogens from clinical specimens using conventional microbial culture and 16S metagenomics: a comparative study. BMC Infect Dis. 2017 Dec 19;17(1):631.

17. Couto N, Schuele L, Raangs EC, Machado MP, Mendes Cl, Jesus TF, et al. Critical steps in clinical shotgun metagenomics for the concomitant detection and typing of microbial pathogens. Sci Rep. 2018 Dec 13;8(1):13767.

18. Zhang M, Hill JE, Alexander TW, Huang Y. The nasal viromes of cattle on arrival at western Canadian feedlots and their relationship to development of bovine respiratory disease. Transbound Emerg Dis. 2020;0-2.

19. Zhang M, Huang Y, Godson DL, Fernando C, Alexander TW, Hill JE. Assessment of metagenomic sequencing and qPCR for detection of influenza D virus in bovine respiratory tract samples. Viruses. 2020;12(8).

20. Gaeta NC, Lima SF, Teixeira AG, Ganda EK, Oikonomou G, Gregory L, et al. Deciphering upper respiratory tract microbiota complexity in healthy calves and calves that develop respiratory disease using shotgun metagenomics. J Dairy Sci. 2017 Feb 1;100(2):1445-58.

21. Klima CL, Holman DB, Ralston BJ, Stanford K, Zaheer R, Alexander TW, et al. Lower Respiratory Tract Microbiome and Resistome of Bovine Respiratory Disease Mortalities. Microb Ecol. 2019 Aug 27;78(2):446-56.

22. Lim A, Naidenov B, Bates H, Willyerd K, Snider T, Couger MB, et al. Nanopore ultra-long read sequencing technology for antimicrobial resistance detection in Mannheimia haemolytica. J Microbiol Methods. 2019 Apr 1;159(August 2018):138-47.

23. Booker CW. Bovine respiratory disease treatment failure: definition and impact. Anim Heal Res Rev. 2020 Dec 8;21(2):172-4.

24. McMullen C, Alexander TW, Léguillette R, Workentine M, Timsit E. Topography of the respiratory tract bacterial microbiota in cattle. Microbiome. 2020;8(1):1-15.

25. Timsit E, Workentine M, Schryvers AB, Holman DB, van der Meer F, Alexander TW. Evolution of the nasopharyngeal microbiota of beef cattle from weaning to 40 days after arrival at a feedlot. Vet Microbiol. 2016 May 1;187:75-81.

26. Lima SF, Teixeira AG V., Higgins CH, Lima FS, Bicalho RC. The upper respiratory tract microbiome and its potential role in bovine respiratory disease and otitis media. Sci Rep. 2016;6(June):1-12.

27. McDaneld TG, Kuehn LA, Keele JW. Evaluating the microbiome of two sampling locations in the nasal cavity of cattle with bovine respiratory disease complex (BRDC). J Anim Sci. 2018;96(4):1281-7.

28. Gould S, Dewell R, Tofflemire K, Whitley RD, Millman ST, Opriessnig T, et al. Randomized blinded challenge study to assess association between Moraxella bovoculi and Infectious Bovine Keratoconjunctivitis in dairy calves. Vet Microbiol. 2013 May 31;164(1-2):108-15. 
29. Dickey AM, Loy JD, Bono JL, Smith TPL, Apley MD, Lubbers B V., et al. Large genomic differences between Moraxella bovoculi isolates acquired from the eyes of cattle with infectious bovine keratoconjunctivitis versus the deep nasopharynx of asymptomatic cattle. Vet Res 2016 471. 2016 Feb 13;47(1):111.

30. Howard CJ, Gourlay RN, Thomas LH, Stott EJ. Induction of pneumonia in gnotobiotic calves following inoculation of Mycoplasma disparand ureaplasmas (T-mycoplasmas). Res Vet Sci. 1976 Sep 1;21(2):227-31.

31. Dabo SM, Taylor JD, Confer AW. Pasteurella multocida and bovine respiratory disease. Anim Heal Res Rev. 2007 Dec 24;8(2):129-50.

32. Thomas AC, Bailey M, Lee MRF, Mead A, Morales-Aza B, Reynolds R, et al. Insights into Pasteurellaceae carriage dynamics in the nasal passages of healthy beef calves. Sci Rep. 2019 Dec 16;9(1):11943.

33. Gagea MI, Bateman KG, Shanahan RA, Van Dreumel T, McEwen BJ, Carman S, et al. Naturally occurring Mycoplasma bovis-associated pneumonia and polyarthritis in feedlot beef calves. J Vet Diagn Invest. 2006;18(1):29-40.

34. Bürki S, Frey J, Pilo P. Virulence, persistence and dissemination of Mycoplasma bovis. Vet Microbiol. 2015 Aug;179(1-2):15-22.

35. Van Donkersgoed J, Ribble CS, Boyer LG, Townsend HG. Epidemiological study of enzootic pneumonia in dairy calves in Saskatchewan. Can J Vet Res. 1993 Oct;57(4):247-54.

36. Virtala AM, Mechor GD, Gröhn YT, Erb HN, Dubovi EJ. Epidemiologic and pathologic characteristics of respiratory tract disease in dairy heifers during the first three months of life. J Am Vet Med Assoc. 1996 Jun 15;208(12):2035-42.

37. Härtel H, Nikunen S, Neuvonen E, Tanskanen R, Kivelä S-L, Aho P, et al. Viral and Bacterial Pathogens in Bovine Respiratory Disease in Finland. Acta Vet Scand. 2004 Dec 1;45(4):193-200.

38. Bottinelli M, Merenda M, Gastaldelli M, Picchi M, Stefani E, Nicholas RAJ, et al. The pathogen Mycoplasma dispar Shows High Minimum Inhibitory Concentrations for Antimicrobials Commonly Used for Bovine Respiratory Disease. Antibiotics. 2020 Jul 29;9(8):460.

39. Ellis JA. The Immunology of the Bovine Respiratory Disease Complex. Vet Clin North Am Food Anim Pract. 2001 Nov;17(3):535-50.

40. Mosier DA. Bacterial Pneumonia. Vet Clin North Am Food Anim Pract. 1997 Nov;13(3):483-93.

41. Johnson JS, Spakowicz DJ, Hong B-Y, Petersen LM, Demkowicz P, Chen L, et al. Evaluation of 16S rRNA gene sequencing for species and strain-level microbiome analysis. Nat Commun. 2019 Dec 6;10(1):5029.

42. Zaheer R, Lakin SM, Polo RO, Cook SR, Larney FJ, Morley PS, et al. Comparative diversity of microbiomes and Resistomes in beef feedlots, downstream environments and urban sewage influent. BMC Microbiol. 2019;19(1):1-17.

43. Klima CL, Zaheer R, Cook SR, Booker CW, Hendrick S, Alexander TW, et al. Pathogens of bovine respiratory disease in North American feedlots conferring multidrug resistance via integrative conjugative elements. J Clin Microbiol. 2014;52(2):438-48.

44. Kanwar N, Scott HM, Norby B, Loneragan GH, Vinasco J, McGowan M, et al. Effects of Ceftiofur and Chlortetracycline Treatment Strategies on Antimicrobial Susceptibility and on $\operatorname{tet}(A)$, tet( $B)$, and blaCMY-2 Resistance Genes among $E$. coli Isolated from the Feces of Feedlot Cattle. PLoS One. 2013 Nov 19;8(11):e80575.

45. Beukers AG, Zaheer R, Cook SR, Chaves A V., Ward MP, Tymensen L, et al. Comparison of antimicrobial resistance genes in feedlots and urban wastewater. Can J Vet Res. 2018 Jan 1;82(1):24

46. Peak N, Knapp CW, Yang RK, Hanfelt MM, Smith MS, Aga DS, et al. Abundance of six tetracycline resistance genes in wastewater lagoons at cattle feedlots with different antibiotic use strategies. Environ Microbiol. 2007 Jan;9(1):143-51.

47. Chaslus-dancla E, Lesage-descauses MC, Leroy-sétrin S, Martel JL, Lafont JP. Tetracycline resistance determinants, TetB and TetM, detected in Pasteurella haemolytica and Pasteurella multocida from bovine herds. J Antimicrob Chemother. 1995 Nov;36(5):815-9.

48. Holman DB, Yang W, Alexander TW. Antibiotic treatment in feedlot cattle: A longitudinal study of the effect of oxytetracycline and tulathromycin on the fecal and nasopharyngeal microbiota. Microbiome. 2019;7(1):1-14.

49. Holman DB, Klima CL, Ralston BJ, Niu YD, Stanford K, Alexander TW, et al. Metagenomic sequencing of bronchoalveolar lavage samples from feedlot cattle mortalities associated with bovine respiratory disease. Genome Announc. 2017;5(40):1-2.

50. Klima CL, Cook SR, Zaheer R, Laing C, Gannon VP, Xu Y, et al. Comparative genomic analysis of Mannheimia haemolytica from bovine sources. PLoS One. 2016;11(2):1-23.

51. Michael GB, Kadlec K, Sweeney MT, Brzuszkiewicz E, Liesegang H, Daniel R, et al. ICEPmu1, an integrative conjugative element (ICE) of Pasteurella multocida: analysis of the regions that comprise 12 antimicrobial resistance genes. J Antimicrob Chemother. 2012 Jan 1;67(1):84-90.

52. Cameron A, Zaheer R, McAllister TA. Emerging Variants of the Integrative and Conjugant Element ICEMh1 in Livestock Pathogens: Structural Insights, Potential Host Range, and Implications for Bacterial Fitness and Antimicrobial Therapy. Front Microbiol. 2019;10(November):1-12.

53. Kinnear A, McAllister TA, Zaheer R, Waldner M, Ruzzini AC, Andrés-Lasheras S, et al. Investigation of Macrolide Resistance Genotypes in Mycoplasma bovis Isolates from Canadian Feedlot Cattle. Pathogens. 2020 Jul 30;9(8):622.

54. Sulyok KM, Kreizinger Z, Wehmann E, Lysnyansky I, Bányai K, Marton S, et al. Mutations Associated with Decreased Susceptibility to Seven Antimicrobial Families in Field and Laboratory-Derived Mycoplasma bovis Strains. Antimicrob Agents Chemother. 2017 Feb 28;61(2):1-14.

55. Lysnyansky I, Ayling RD. Mycoplasma bovis: Mechanisms of resistance and trends in antimicrobial susceptibility. Front Microbiol. $2016 ; 7(A P R): 1-7$.

56. Nayfach S, Rodriguez-Mueller B, Garud N, Pollard KS. An integrated metagenomics pipeline for strain profiling reveals novel patterns of bacterial transmission and biogeography. Genome Res. 2016 Nov 1;26(11):1612-25.

57. Bell CJ, Blackburn P, Elliott M, Patterson TIAP, Ellison S, Lahuerta-Marin A, et al. Investigation of polymerase chain reaction assays to improve detection of bacterial involvement in bovine respiratory disease. J Vet Diagnostic Investig. 2014;26(5):631-4. 
58. CLSI. Performance Standards for Antimicrobial Disc and Dilution Susceptibility Tests for Bacterial Isolated from Animals. Clinical Laboratory Standards Institute; 2020.

59. Jelinski M, Kinnear A, Gesy K, Andrés-Lasheras S, Zaheer R, Weese S, et al. Antimicrobial Sensitivity Testing of Mycoplasma bovis Isolates Derived from Western Canadian Feedlot Cattle. Microorganisms. 2020 Jan 16;8(1):124.

60. Wick RR, Judd LM, Gorrie CL, Holt KE. Completing bacterial genome assemblies with multiplex MinION sequencing. Microb Genomics. 2017;3(10).

61. De Coster W, D'Hert S, Schultz DT, Cruts M, Van Broeckhoven C. NanoPack: Visualizing and processing long-read sequencing data. Bioinformatics. 2018;34(15).

62. Wood DE, Lu J, Langmead B. Improved metagenomic analysis with Kraken 2. Genome Biol. 2019 Dec 28;20(1):257.

63. Hayes BJ, Daetwyler HD. 1000 Bull Genomes Project to Map Simple and Complex Genetic Traits in Cattle: Applications and Outcomes. Vol. 7, Annual Review of Animal Biosciences. 2019.

64. Lu J, Breitwieser FP, Thielen P, Salzberg SL. Bracken: Estimating species abundance in metagenomics data. PeerJ Comput Sci. 2017;2017(1).

65. Ondov BD, Bergman NH, Phillippy AM. Interactive metagenomic visualization in a Web browser. BMC Bioinformatics. 2011;12(September).

66. Seeman T. Abricate. https://github.com/tseemann/abricate;

67. Feldgarden M, Brover V, Haft DH, Prasad AB, Slotta DJ, Tolstoy I, et al. Validating the AMRFINder tool and resistance gene database by using antimicrobial resistance genotype-phenotype correlations in a collection of isolates. Antimicrob Agents Chemother. 2019;63(11).

68. Li H. Minimap2: Pairwise alignment for nucleotide sequences. Bioinformatics. 2018;34(18).

69. Li H, Handsaker B, Wysoker A, Fennell T, Ruan J, Homer N, et al. The Sequence Alignment/Map format and SAMtools. Bioinformatics. 2009;25(16).

70. Altschul SF, Gish W, Miller W, Myers EW, Lipman DJ. Basic local alignment search tool. J Mol Biol. 1990/10/05. 1990;215(3):403-10.

71. Camacho C, Coulouris G, Avagyan V, Ma N, Papadopoulos J, Bealer K, et al. BLAST plus: architecture and applications. BMC Bioinformatics. 2009;10(421):1.

72. Kolmogorov M, Bickhart DM, Behsaz B, Gurevich A, Rayko M, Shin SB, et al. metaFlye: scalable long-read metagenome assembly using repeat graphs. Nat Methods. 2020;17(11).

73. Lin Y, Yuan J, Kolmogorov M, Shen MW, Chaisson M, Pevzner PA. Assembly of long error-prone reads using de Bruijn graphs. Proc Natl Acad Sci U S A. 2016;113(52).

74. Von Meijenfeldt FAB, Arkhipova K, Cambuy DD, Coutinho FH, Dutilh BE. Robust taxonomic classification of uncharted microbial sequences and bins with CAT and BAT. Genome Biol. 2019;20(1).

75. Seeman T. Snippy [Internet]. Available from: https://github.com/tseemann/snippy

\section{Table 3}

Table 3 is available in the Supplementary Files section.

\section{Figures}

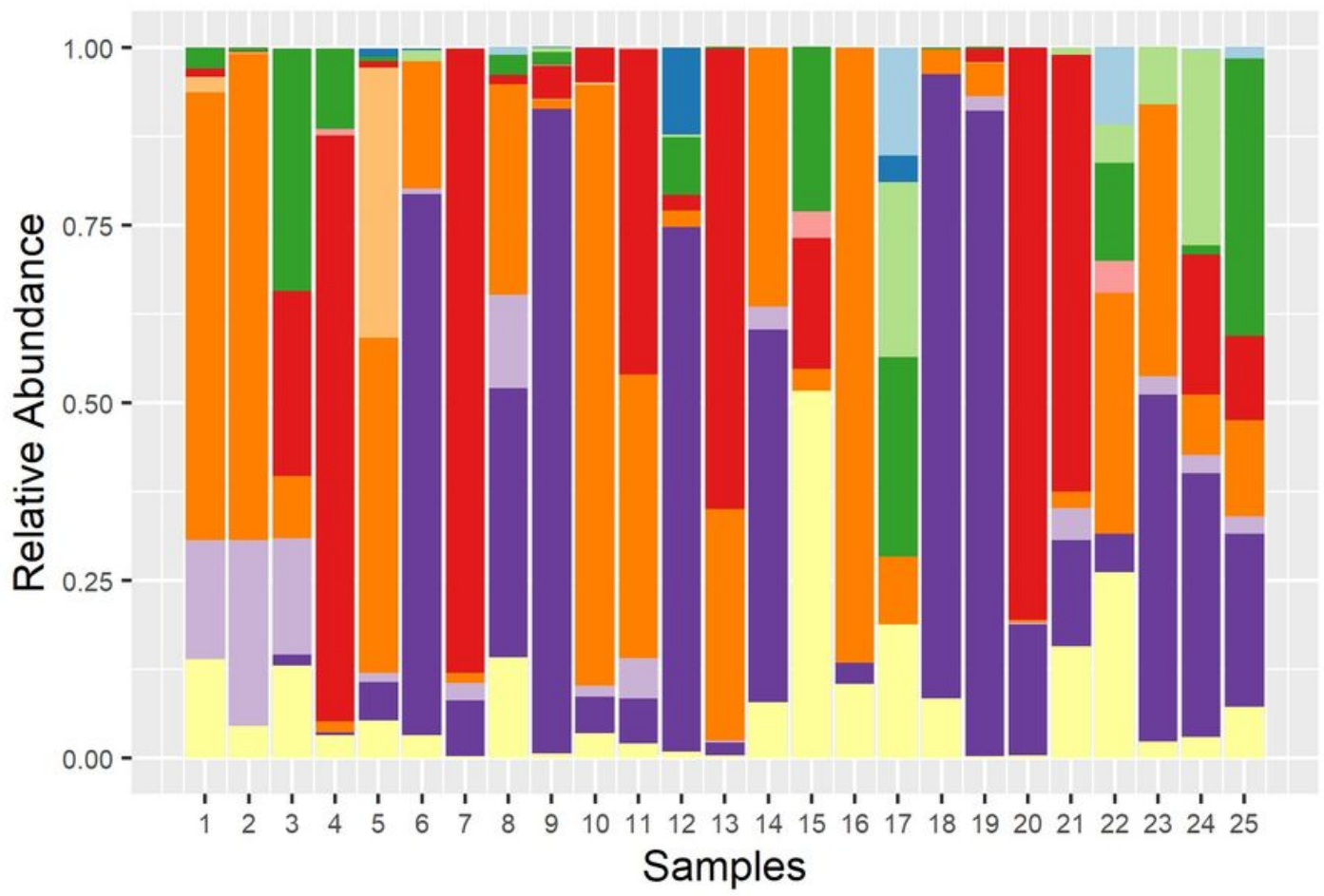

\section{Species}

Bibersteinia trehalosi
Histophilus somni
Mannheimia haemolytica
Mannheimia spp. (other)
Moraxella bovis
Moraxella bovoculi
Mycoplasma bovis
Mycoplasma dispar
Mycoplasma sp. (other)
Pasteurella multocida
Other

Page 12/13 
Relative abundance of bacterial taxa across all samples. Each column represents the relative abundance of non-host sequencing bases attributed to ten taxonomic groups of interest. Bases that were classified as anything other than the ten taxonomic groups listed above were grouped into the "Other" category

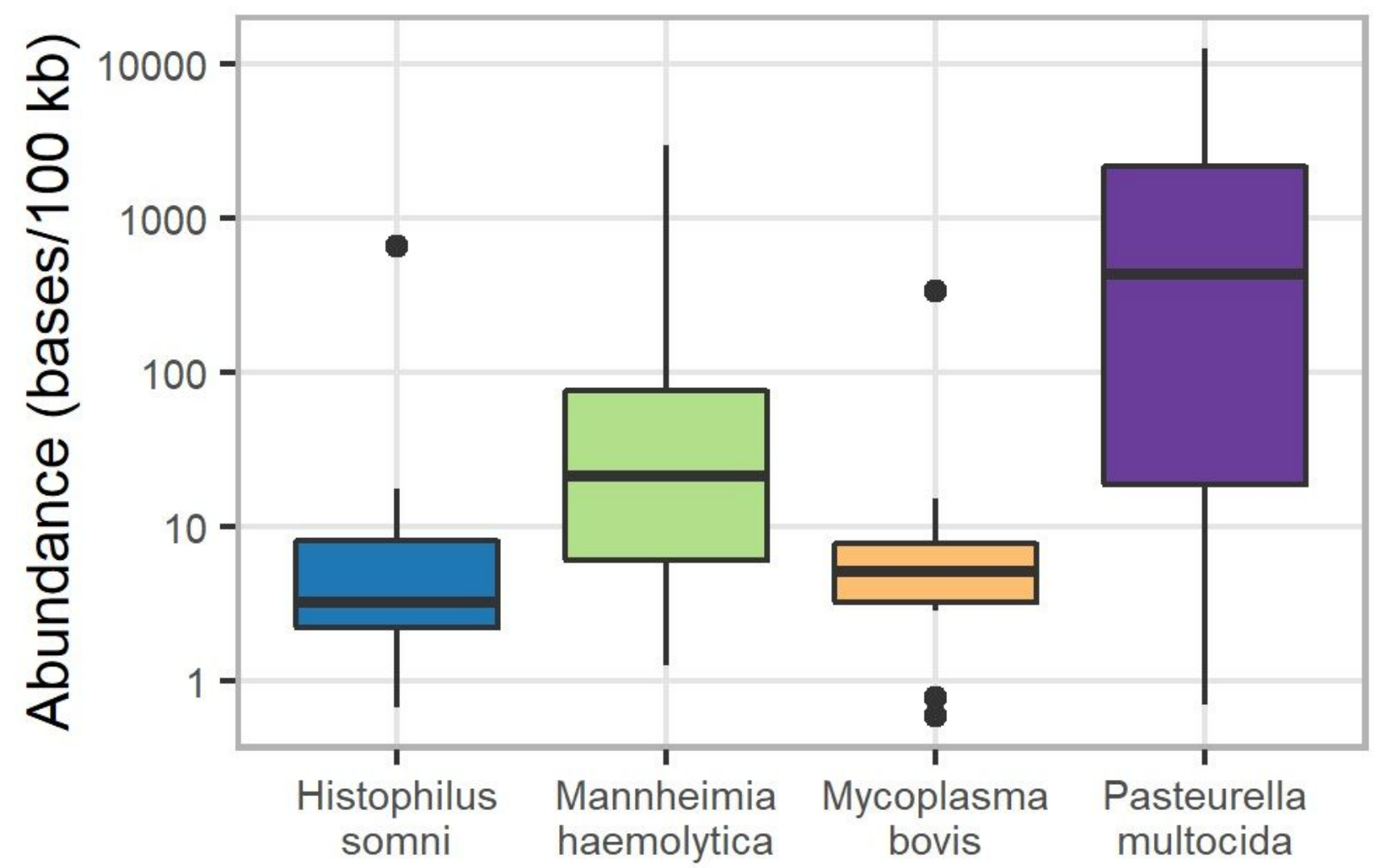

Figure 2

Base-pairs of BRD associated bacteria identified across all samples. The number of bases classified as each of the four target organisms was calculated for each sample and expressed per $100 \mathrm{~kb}$ sequenced. In each boxplot, the middle horizontal line represents the median, and the top and bottom horizontal line represent the 75th and 25th percentile, respectively. Outlier values are indicated as dots and represent any values that are over 1.5 times the interquartile range over the 75th percentile or under the 25th percentile.

\section{Supplementary Files}

This is a list of supplementary files associated with this preprint. Click to download.

- S.Table1.docx

- S.Table2.docx

- Table3.docx 